Technical report DAIMI-PB-569

\title{
Declarative Specialization for Object-Oriented-Program Specialization
}

\author{
Helle Markmann Andersen* Ulrik Pagh Schultz \\ Department of Informatics DAIMI/ISIS \\ County of Aarhus University of Aarhus
}

May 12, 2004

\begin{abstract}
The use of partial evaluation for specializing programs written in imperative languages such as $\mathrm{C}$ and Java is hampered by the difficulty of controlling the specialization process. We have developed a simple, declarative language for controlling the specialization of Java programs, and interfaced this language with the JSpec partial evaluator for Java. This language, named Pesto, allows declarative specialization of programs written in an object-oriented style of programming. The Pesto compiler automatically generates the context information needed for specializing Java programs, and automatically generates guards that enable the specialized code in the right context.
\end{abstract}

Word count (detex I wc): 5039

\section{Introduction}

Partial evaluation is an automated technique for mapping generic programs into specific implementations dedicated to a specific purpose. Partial evaluation has been investigated extensively for functional $[4,5]$, logical [14] and imperative $[2$, $3,6]$ languages, and has recently been investigated for object-oriented languages by Schultz et al. [19, 20, 21].

To specialize a program the user must specify the context for which the program is to be specialized. For imperative languages, not only must the binding times of the entry point parameters be specified, but alias relations and the individual binding times of each heap location of the context must also be specified. A partial evaluator must provide an interface for specifying this information, but use of this interface is typically tedious and error-prone. Worse, the specialized code is only correct in the context it was specialized for, but the

*Based on work done while a student at the University of Aarhus 
decision of when to use the specialized code must by implemented manually by the user. To remedy this problem, Volanschi et al. defined the language specialization classes, that allows the user to declaratively specify the context for which the program should be specialized [24]. When using this language to specify the context, guards are automatically generated that execute the specialized code only in the right context.

Specialization classes were however defined before partial evaluation for object-oriented languages was fully developed. Although conceptually appropriate for controlling the specialization of object-oriented programs, in practice specialization classes suffer from a number of limitations which makes

it difficult to use them to specialize programs written in an object-oriented style $[18,20,21,22]$. Furthermore, the specialization classes compiler is not integrated with a partial evaluator, so manual work is still needed.

We have developed an extended version of the specialization classes language, named Pesto, which addresses the shortcomings of specialization classes. The contributions of this paper are as follows:

- Extension of the syntax and semantics of declarative specialization to support specialization of programs written in an object-oriented style of programming.

- Integration with an existing state-of-the-art partial evaluator for Java: automatic generation of the context used for binding-time analysis, the context used for specialization, and the guards used at run-time to select the specialized code.

- Encapsulation of the residual code and associated guards into a single module, using aspect-oriented programming.

Although Pesto as presented here is integrated with a specific partial evaluator, we believe that the same principles would be usable with other partial evaluators for object-oriented languages.

\section{Specialization classes vs. Pesto}

Our work is based on the concept of declarative specialization, as represented by specialization classes $[23,24]$. Nevertheless, the syntax and semantics has evolved to the extent that we first present Pesto independently of specialization classes, and then afterwards compare Pesto to specialization classes.

\section{Overview}

The rest of this paper is organized as follows. We first cover background and motivation (Section 2), and then Section 3 presents the Pesto language. The compilation of Pesto to a JSpec context is detailed in Section 4, Section 5 experimentally assesses the overhead due to using guards, and Section 6 discusses related work. Last, Section 7 presents our conclusions and outlines future work. 


\section{Background and Motivation: Object-Oriented- Program Specialization}

\subsection{Partial evaluation}

Partial evaluation is a program transformation technique that optimizes a program fragment with respect to information about a context in which it is used, by generating an implementation dedicated to this usage context. Partial evaluation works by aggressive inter-procedural constant propagation of values of all data types [10]. Partial evaluation thus adapts a program to known (static) information about its execution context, as supplied by the user (the programmer). Only the program parts controlled by unknown (dynamic) data are reconstructed (residualized). In this paper, we work with off-line partial evaluation, where a preprocessing phase, the binding-time analysis, separates the program into its dynamic and static parts, after which a specialization phase evaluates the static parts of the program.

\subsection{Partial evaluation for object-oriented languages}

Partial evaluation of an object-oriented program is based on the specialization of its methods [21]. The optimizations performed includes eliminating virtual dispatches with static receivers, reducing imperative computations over static values, and embedding the values of static (known) fields within the program code. A specialized method thus has a less general behavior than the unspecialized method, and it accesses only those parts of its parameters (including the this object) that were considered dynamic.

Typically, an object-oriented program uses multiple objects that interact using virtual calls. For this reason, the specialized methods generated for one class often need to call specialized methods defined in other classes. Thus, partial evaluation of an object-oriented program creates new code with dependencies that tend to cross-cut the class hierarchy of the program. This observation brings aspect-oriented programming to mind; aspect-oriented programming allows logical units that cut across the program structure to be separated from other parts of the program and encapsulated into an aspect [12]. The methods generated by a given specialization of an object-oriented program can be encapsulated into a separate aspect, and only woven into the program during compilation. Access modifiers can be used to ensure that specialized methods only can be called from specialized methods encapsulated in the same aspect, and hence always are called from a safe context. Furthermore, the specialized code is cleanly separated from the generic code, and can be plugged and unplugged by selecting whether to include the aspect in the program.

\section{Example: generic power function}

Figure 1 shows an implementation of the classic power function parameterized by an exponent and a binary operator (which must be a subclass of BinOp). The 


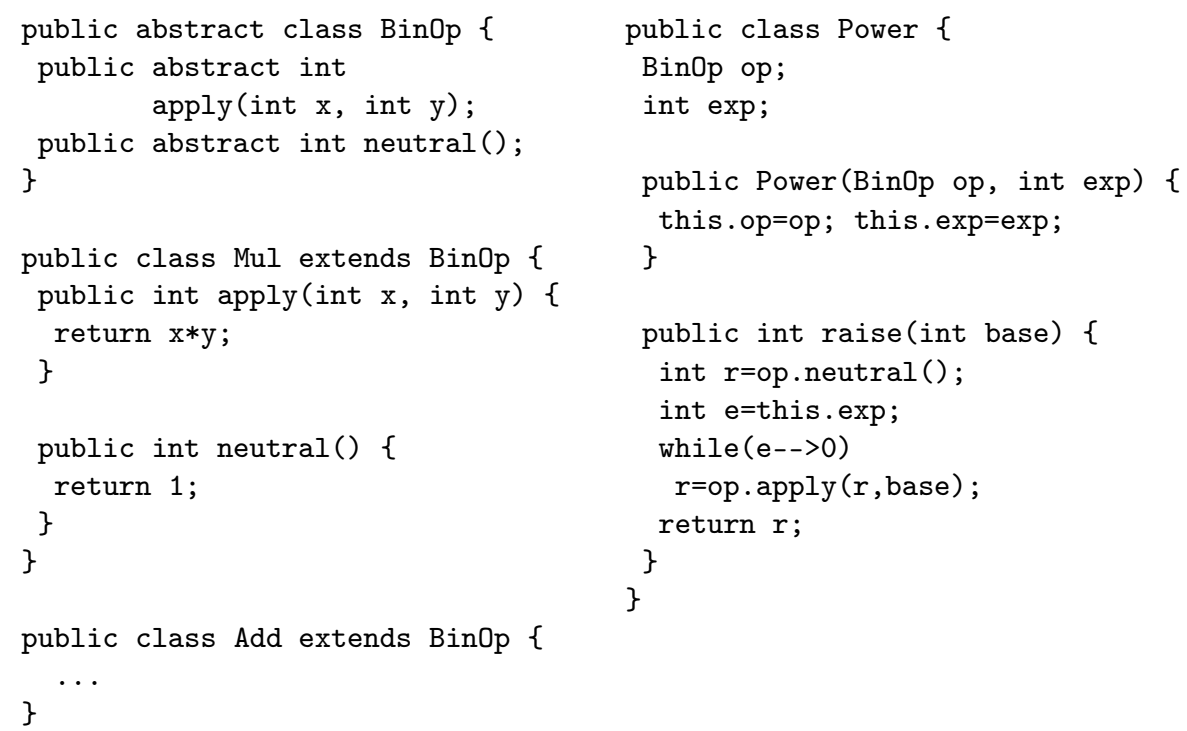

Figure 1: Generic power program

class Power could be used as follows:

(new Power (new Mul ()$, 3)$ ). raise (x)

This expression computes $x^{3}$. We can specialize the method Power. raise for this exponent and operator, to obtain a more efficient version. The result is shown in Figure 2, in AspectJ [11, 25] syntax. The aspect lists two methods to introduce into the classes of the program. The method raise_spec is public and is therefore visible outside the aspect. In this method, the loop has been unrolled, and a virtual dispatch is no longer needed when invoking the binary operator. The method apply_o is private to the aspect and is therefore only visible to other methods defined within the same aspect. ${ }^{1}$ When the programmer knows that the cubic operation is needed, the method raise_spec can simply be called on an instance of class Power.

\subsection{The JSpec partial evaluator}

JSpec is an off-line partial evaluator for the Java language, excluding exception handlers, multithreading, reflection and finally regions. It takes as input Java bytecode and native functions, and generates residual code in AspectJ $[11,25]$ syntax. The JSpec binding-time analysis is context-sensitive,

\footnotetext{
${ }^{1}$ The method apply_0 could in this case easily be inlined into the caller. Nevertheless, in general methods cannot always be inlined between classes, because they may need to access private members in the residual program. Moreover, in the case of Java, inlining is often best left to the dynamic compiler [21]. For these reasons, we do not use method inlining in this paper.
} 


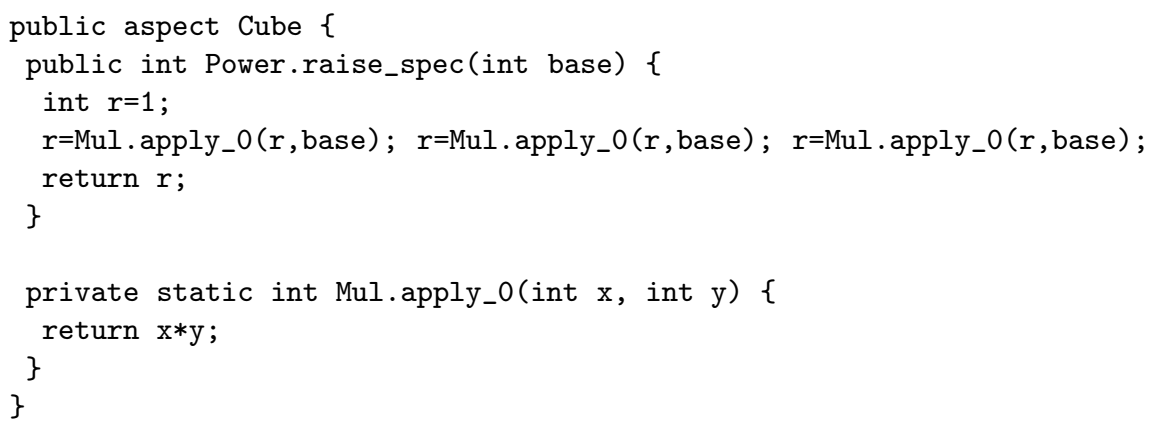

Figure 2: Power program specialized for three multiplications

class-polyvariant (each object creation site is assigned a binding time individually), use-sensitive [8], and flow-sensitive. JSpec is applied to a user-selected program slice, which allows time-consuming analyses and aggressive transformations to be directed towards critical parts of the program.

JSpec is nonetheless not an easy tool to use. A major usability issue is the way the programmer interacts with the partial evaluator. To specialize a program slice, the user is required to describe the context of this slice to the partial evaluator. For Java, the context includes information on the binding time and alias relation of the parameters of the method to be specialized, including the this object and any objects it may refer to. This information is communicated to the partial evaluator by writing a piece of code that computes the context for which the method is to be specialized. This approach allows an arbitrary context to be created, but is often more general than what is needed. Moreover, programming the context correctly is difficult, even for an expert programmer.

\section{Example: the context of the power function}

A general context which allows JSpec to specialize the power program of Figure 1 for any exponent and any of the two binary operators is shown in Figure 3. The class AnalysisContext defines a static field for each of the parameters of the entry point and the static method set initializes these fields to the context required for analysis. This method uses a static conditional to select which operator to use. This idiom indicates to the partial evaluator that the operator is statically known and is either of class Mul or class Add. The class SpecializationContext is similarly constructed, but reads the concrete choice of operator and exponent from a file. Reading this information from a file avoids having to recompile the specializer every time the context changes. Both these classes must however be written by the user, which is tedious and error-prone. 


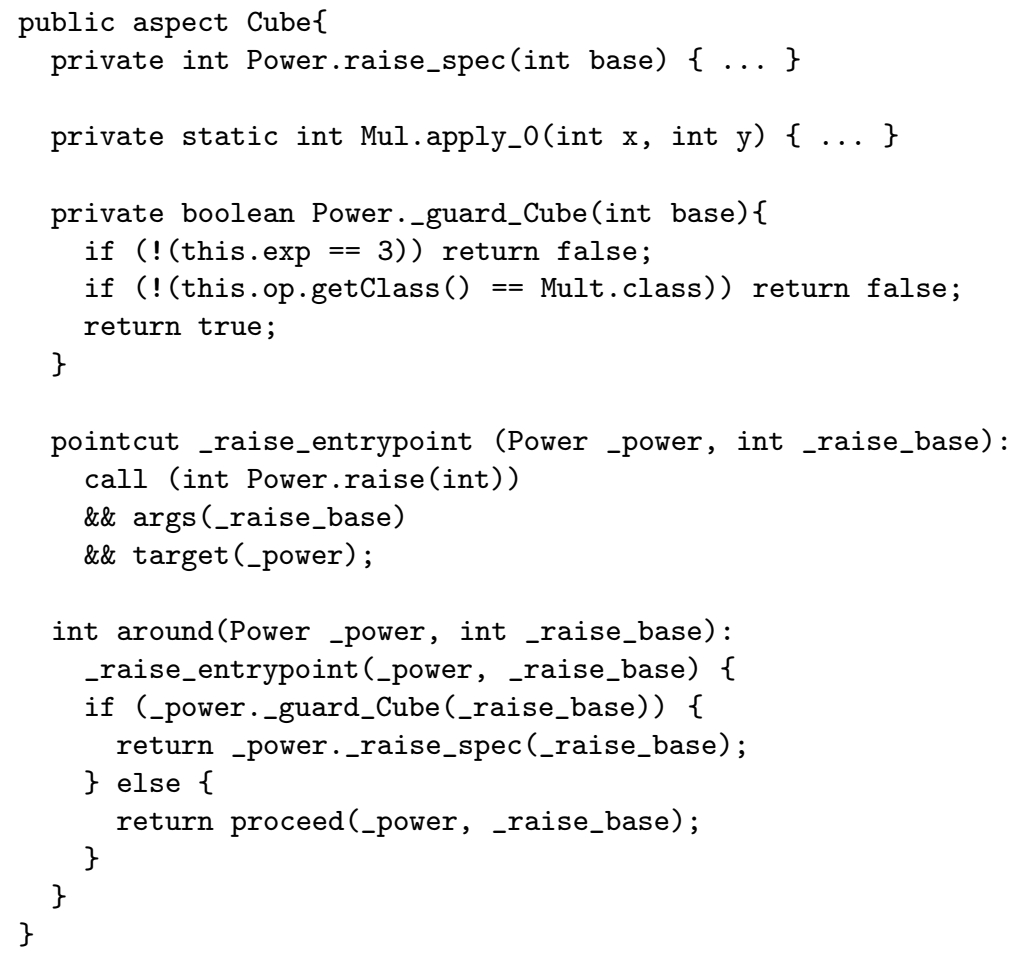

Figure 5: Specialized program generated by the Pesto compiler for the Cube specialization class

specialization process. These are communicated to Pesto using a values file which contains the concrete values for a specific scenario.

\subsection{Predicates on fields}

A fixed static predicate on a primitive field $\mathrm{x}$ can be declared as: " $\mathrm{x}==3$;", indicating that in this scenario $x$ always has the value 3 . A static predicate for the same field is simply declared as: " $\mathrm{x}==$ !;". Conversely, a fixed static predicate on a reference field y is written: "y: c;". This indicates that the field y references an object of class C. Specifically, the predicate is true when the concrete type of the object referred to by y is C; subclasses of C are not allowed. A static predicate on a field indicates the set of possible classes that the field may refer to: "y: C1 । . . । Cn;". To express predicates on an object referred to by a field, the field is qualified by a specialization class rather than a Java class, e.g. "y: c;" meaning that y must fulfill the predicates declared in the specialization class $\mathrm{C}$. 


\subsection{Entry point declaration}

The entry point is the method that is targeted by the specialization process, along with any callees defined in other classes of the specialization module. The entry point specifies a method signature in standard Java syntax, but can optionally also specify predicates on the formal parameters. For example:

int raise (int base) $\{$ where base $==2$; $\}$

To specify that the Power.raise method is to be specialized with 2 as the base value. The same predicates as were usable on fields are usable on formal parameters, with identical syntax.

\subsection{Arrays}

Predicates over references to arrays can be used to specify the type of the array object, the length of the array, and the contents the array. The predicate " $\mathrm{x}$ : Power [!]" indicates that the variable $\mathrm{x}$ refers to an array of type Power [] with a statically known length (a fixed length could also have been specified). The contents of a fixed static array can be specified as follows:

$$
\mathrm{x}: \operatorname{BinOp}[2]=\{\mathrm{Mul}, \text { Add }\} \text {; }
$$

This predicate indicates that $\mathrm{x}$ references an array of size 2 where the first element has concrete type Mul and the second element has concrete type Add. Similarly, the contents of a static array can be specified as follows:

$$
\mathrm{x}: \operatorname{BinOp}[!]=[\text { Mul | Add }] \text {; }
$$

This predicate indicates that $\mathrm{x}$ references an array with static length, and that the objects contained in the array have concrete types Mul or Add. As was the case earlier, specialization classes can be used in the place of concrete classes to specify invariants over the fields of the objects contained in the array.

\subsection{Declaring alias information}

Alias information is a critical part of the program context, since side-effects are traced using an alias analysis. The semantics of Pesto is that each specialization class represents one or more objects of the Java class that is specialized by the specialization class. These objects are assumed to be aliased, whereas the sets of objects represented by other specialization classes are assumed to be disjoint from this set. Likewise, predicates that qualify a reference by a Java class are assumed to refer to disjoint sets of objects.

The specialization phase operates on a concrete context where object instances are manipulated by the static code parts. These object instances must correspond to the concrete instances from the context, so all aliasing must be disambiguated. This is done in the values file, by annotating the specialization class instantiations with variant numbers. For example, Mul\#1 and Mul\#2 denotes 


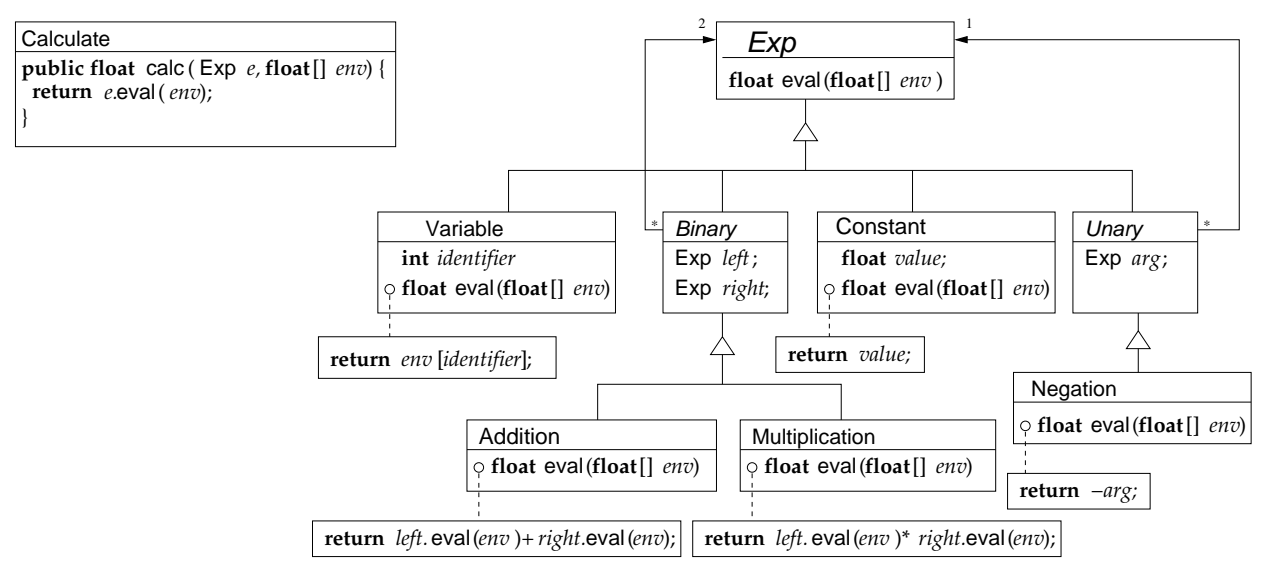

Figure 6: Class diagram of arithmetic expression interpreter

two different instances of the class Mul. Instances without variant numbers are assigned the default number zero.

A guard that unambiguously recognizes a specific aliasing context must compare the identity of all objects in the context, for example to determine if two fields that were declared to refer to different objects refer to the same object. Performing such a comparison is non-trivial and is computationally expensive (quadratic in the number of objects). Nonetheless, precise treatment of aliases is not always required, so the programmer should be allowed to select between precise and approximate guarding with regards to alias information. The current implementation of Pesto only supports approximate guarding, where the fields of each object are inspected, but the identity of each object is not tested.

\section{Example: arithmetic expression interpreter}

As an example, we use the arithmetic expression interpreter summarized in the class diagram of Figure 6. Here, the recursive method eval is defined by each class, and takes an environment that maps each variable (numbered by an integer) to its value. The method calc can be specialized for a concrete expression, to produce a compiled Java expression.

An excerpt of the required specialization module is shown in Figure 7. The specialization class SpecExp describes the entry point. Each type of node is specialized by a specific specialization class with static references to all other kinds of nodes, describing a mutually recursive data structure. The values file shown in Figure 8 contains the instantiated specialization classes for the expression " $87 *(x * x)$ ". The two multiplication nodes are represented as separate objects, as are the objects of class Variable. Nevertheless, the guards which Pesto generates for this expression cannot differentiate between representing this expression as a tree and as a DAG. In this particular case, since there are no side-effects on 


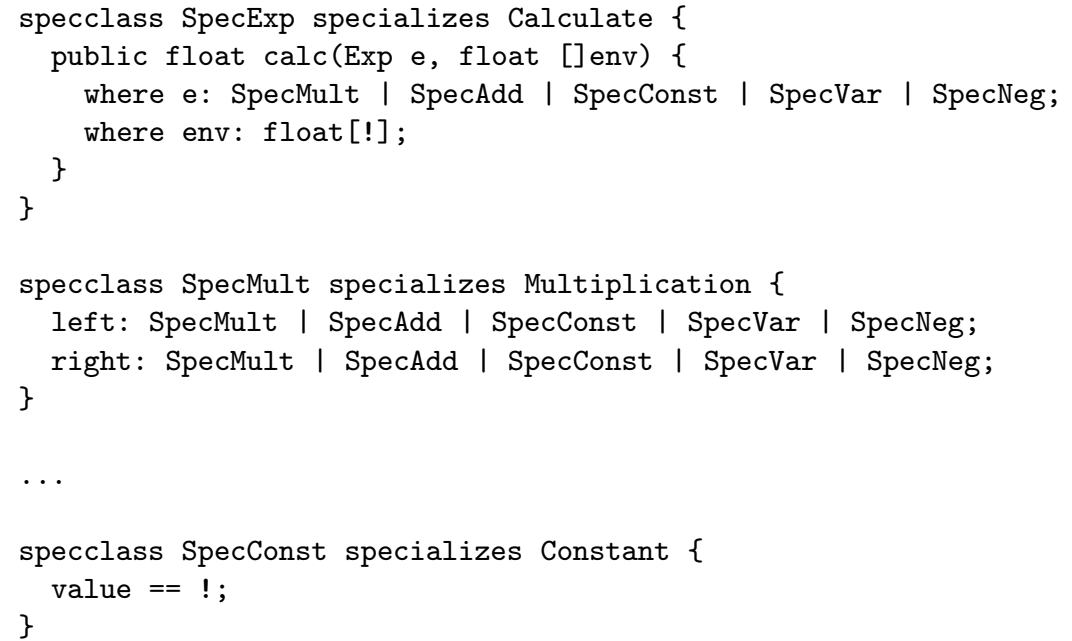

Figure 7: Specialization module for the program of Figure 6

the nodes and no object identity comparisons, both a tree context and a DAG context is correct for the specialized code.

\section{Compiling Pesto}

We describe the compilation of a simplified version of Pesto to Java by a syntaxdirected translation. Pesto supports all Java primitive types and arrays; the simplified version of Pesto that we describe only supports integer as a primitive type and does not support arrays. The compilation of predicates involving arrays are described informally at the end of this section.

\subsection{Overview}

Compilation of a specialization module generates the analysis context, specialization context, and values file template. The guards are generated by the specialization context, when the concrete specialization context has been computed. The guards are combined with the output of JSpec to form a complete residual program. Compilation also generates a configuration file based on options declared directly in the specialization module; we refer to the first author's MS for details [1].

The current version of Pesto uses no-argument constructors to create object instances and directly assigns values to fields. Thus, any class which is to be specialized must contain a no-argument constructor and must declare its fields as public (these limitations are however not intrinsic to Pesto, as described in Section 7). 


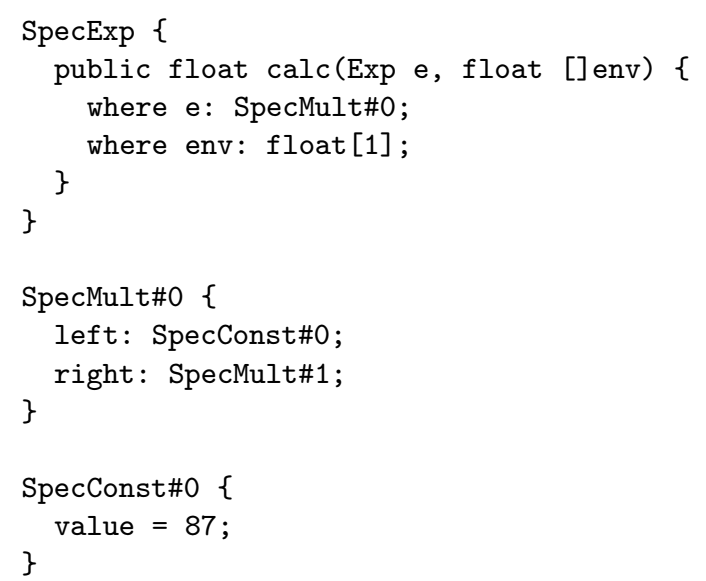

Figure 8: Values file containing instantiated specialization classes for the scenario described in Figure 7

\subsection{Analysis context}

Figure 9 shows the top-level translation for the analysis context. The first rule takes a specialization class $E$ containing an entry point and a number of specialization classes, $S_{1}, \ldots, S_{m}$. They are translated to the class AnalysisContext which contains the method set. The rule $\mathcal{D}$ declares the fields required by JSpec, e.g., _this and the parameters of the entry point. The rule $\mathcal{I}$ produces a declaration of an object instance for each specialization class. In the JSpec binding-time analysis, an object allocation site is interpreted as producing one or more objects, which matches the semantics of Pesto. To produce the required context, assignments are made to the fields of these object instances.

Figure 10 shows the assignment of binding times and alias relations for predicates; the rule lhs (left hand side) abstracts over whether a variable is an instance variable or a parameter. The methods in the classes StaticValue and DynamicValue are provided by the JSpec environment, and are used to obtain static and dynamic values, respectively. The method DynamicValue.get_object() represents an allocation site which can produce an object of any class used in the program (the set of all classes is known when the partial evaluator processes the program).

A predicate on a reference qualified by a Java type $J$ is handled by assigning a new object of class $J$, which gives the field static binding time and sets the alias relation to this object. A static predicate uses the static conditional idiom to associate the variable with the set of possible classes. The rule for " $v: S$ " handles fixed static predicates that bind a variable to a specialization class by assigning the variable to the corresponding instance that represents the specialization class. 


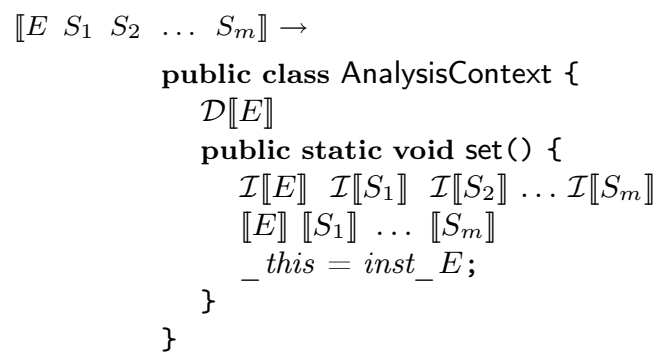

$\mathcal{D} \llbracket$ specclass $E$ specializes $J\left\{\begin{array}{lllll}p_{1} & p_{2} & \ldots & p_{k} & e\end{array}\right\} \rrbracket \rightarrow$ public static $J$ this; $\mathcal{D} \llbracket e \rrbracket$

$\mathcal{D} \llbracket$ public int $f\left(T_{1} p_{1}, T_{2} p_{2}, \ldots, T_{h} p_{h}\right)$ \{where $p_{1} \ldots$ where $\left.p_{k}\right\} \rrbracket \rightarrow$ public static $T_{1} p_{1}$;

$\vdots$

public static $T_{h} p_{h}$;

$\mathcal{I} \llbracket$ specclass $N$ specializes $J\left\{\begin{array}{llllll}p_{1} & p_{2} & \ldots & p_{k} & e\end{array}\right\} \rrbracket \rightarrow J$ inst_ $N=$ new $J()$;

Figure 9: Top-level declarations for the analysis context

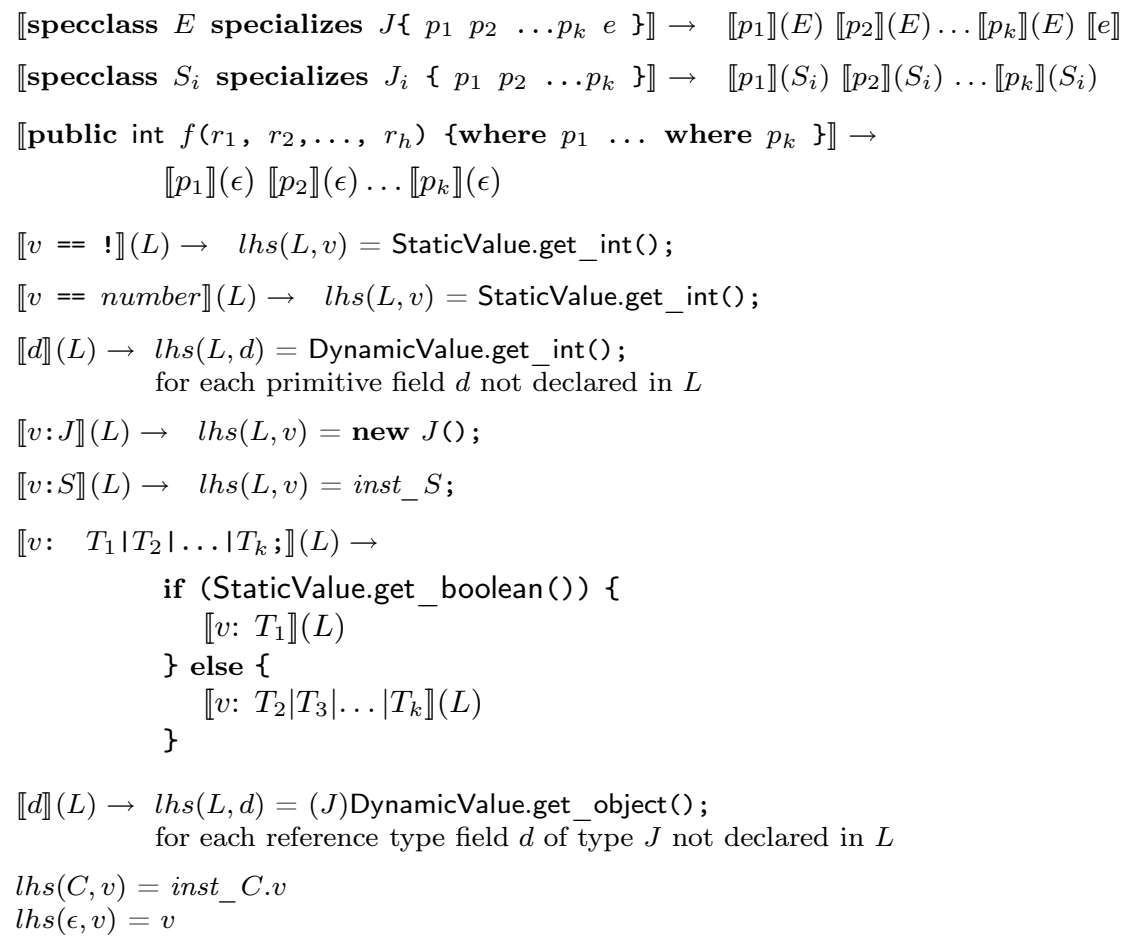

Figure 10: Translation of predicates for the analysis context 


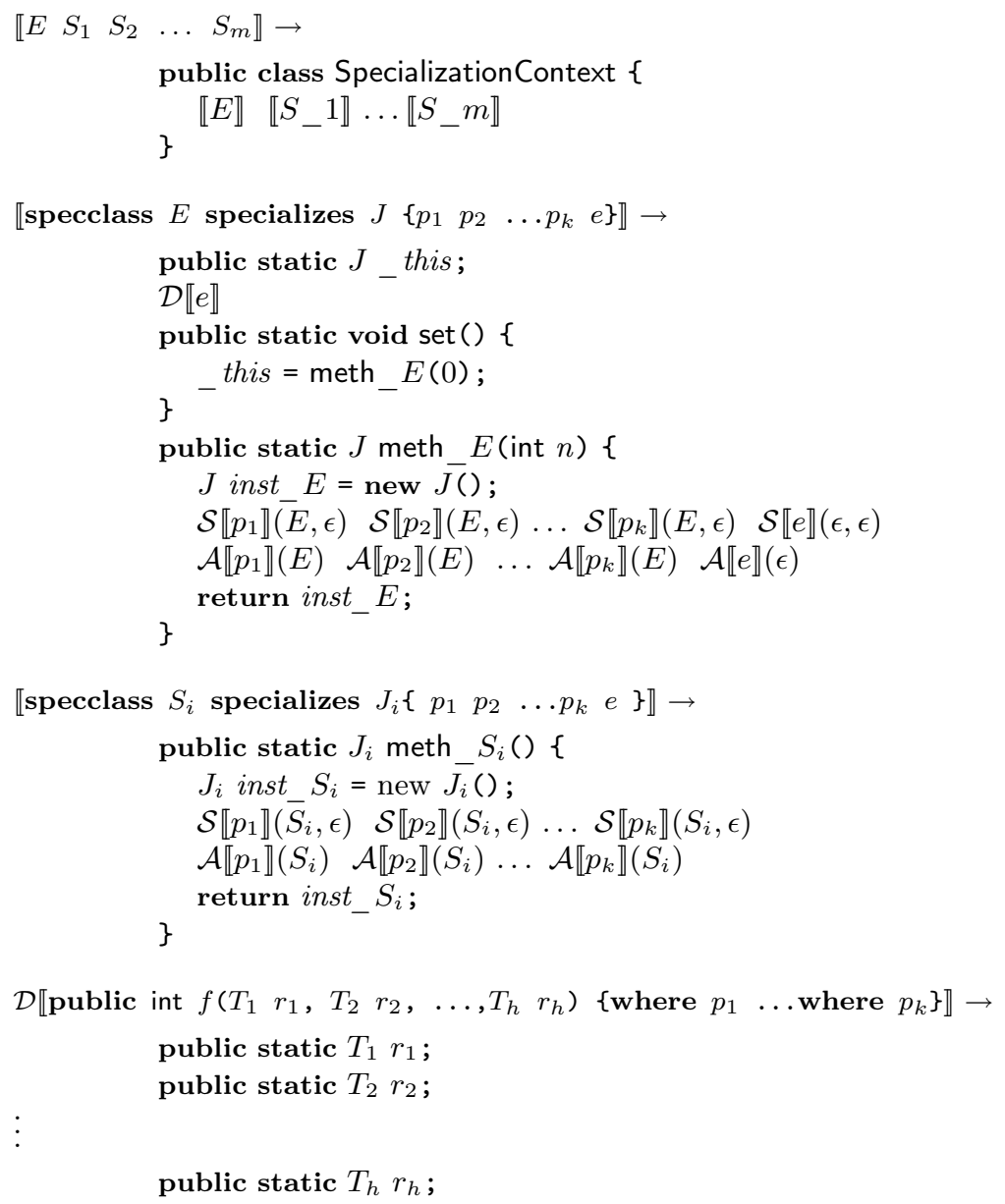

Figure 11: Top-level declarations for the specialization context

\subsection{Specialization context}

The specialization context creates the concrete context for which the program slice is to be specialized. The values file containing the concrete specialization class instantiations is parsed during specialization and used to compute the context. Generation of guards is performed by the code generated for the specialization context, but we for clarity present this separately.

The translation from declarations to specialization context is shown in Figure 11. For each specialization class in the file, a method is generated which creates and initializes an instance of this specialization class; it takes as argument an integer which indicates the variant to generate (see Section 3.6). The generation of each predicate uses two rules, $\mathcal{S}$ and $\mathcal{A}$. The rule $\mathcal{S}$ generates code to obtain the concrete value either implicitly from the specialization class 


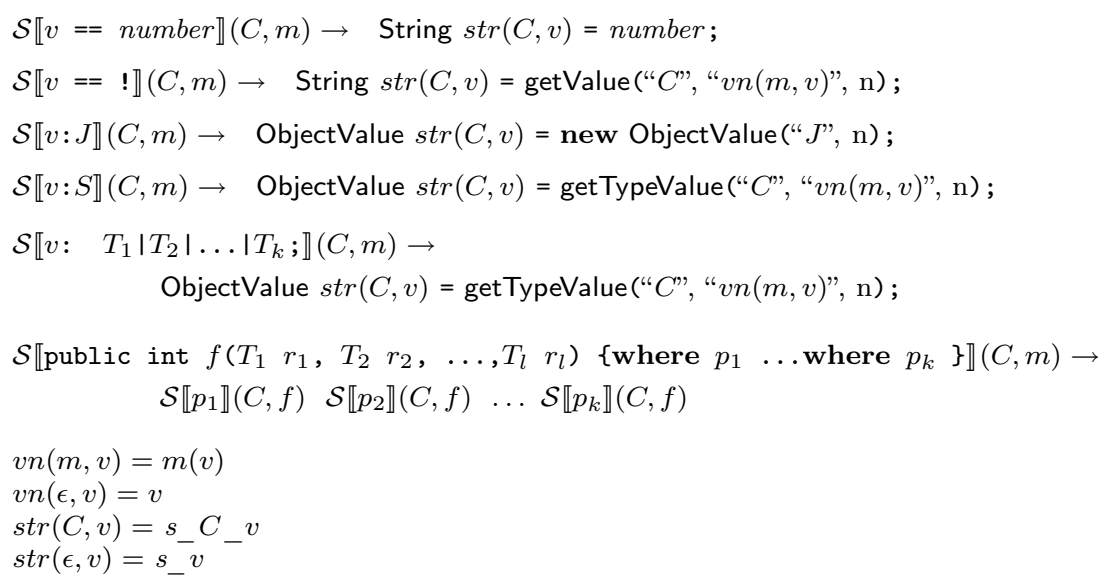

Figure 12: Collecting concrete values in the specialization context

(fixed static predicate) or by reading it from the values file. This rule uses two arguments: the name of the specialization class and the name of the method if the predicate concerns the entry point. The rule $\mathcal{A}$ assigns the value just obtained to the appropriate variable.

The definition of $\mathcal{S}$ is shown in Figure 12. For a fixed static predicate, the value is stored in the variable denoted by $\operatorname{str}(C, v)$. Otherwise, the method getvalue is called with the name of the specialization class, the name of the variable, and the variant number as arguments; this method reads the concrete value from the values file. The same construction is used for reference types, except that the value is stored in an objectValue object.

After collecting the concrete values, they are assigned to the appropriate variables, as shown in Figure 13. In general, the value is obtained from the variable created in $\mathcal{S}, \operatorname{str}(C, v)$. The rule " $v: S$ " assigns a value by recursively calling the method meth_S with the specialization class variant number as an additional argument. The rule " $v: T_{1}\left|T_{2}\right| \ldots \mid T_{n}$ " generates code to recursively call the correct method depending on the value read from the values file.

\subsection{Guards}

Guards are used at runtime to select the specialized entry point when it is called from the context for which it was specialized. The concrete context which was computed by the method specializationContext.set during specialization is used to generate the guards, so all predicates can be treated as if they were fixed static. The guards are encapsulated into the same aspect as the specialized code, and are introduced as private methods into the concrete Java classes they need to inspect.

There are primarily two kinds of guards: call-time guards which check the entire context when the entry point is called and modification-time guards which 


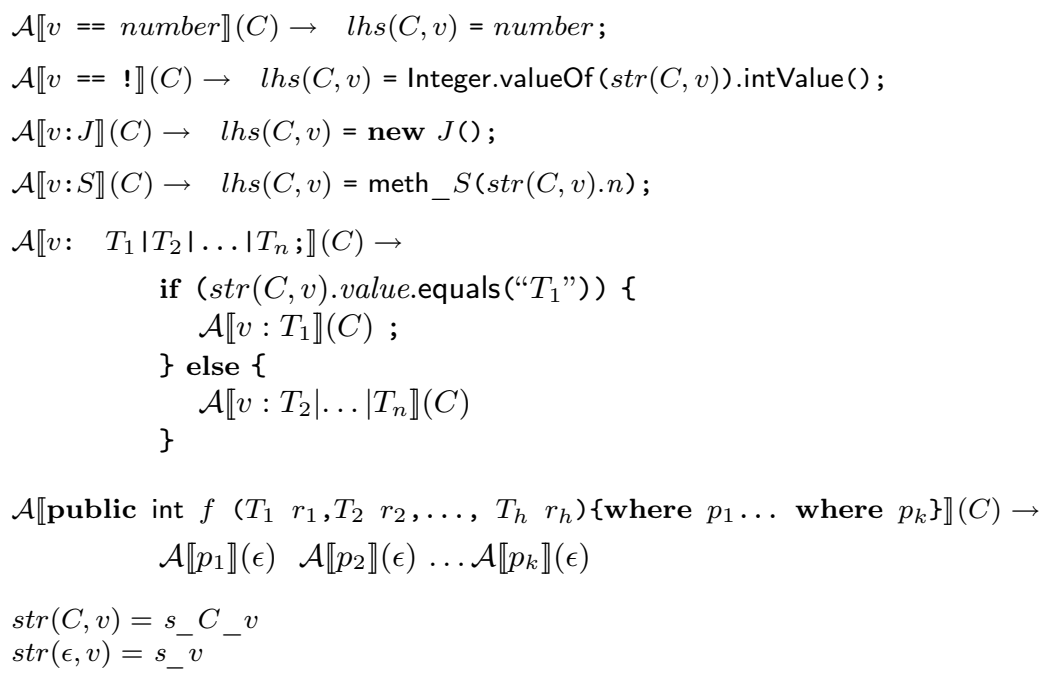

Figure 13: Assigning the concrete values in the specialization phase.

incrementally check the context every time a field is modified. Depending on the scenario, modification-time guards may be more efficient than call-time guards, but are more limited: only private fields can safely be guarded. Since arrays effectively only contain public fields (the length and the contents), modificationtime guards cannot be used to protect them. Moreover, predicates on entry point parameters are only meaningful with call-time guards. Pesto only implements call-time guards; we consider the support for modification-time guards to be future work.

The specialization module is translated into an aspect where the specialized methods must be inserted. Each specialization class is translated into a guard method, as shown in Figure 14. The rule $\mathcal{G}$ shown in Figure 15 generates the code needed to check each predicate. All predicates must be true for the guard to be satisfied.

\subsection{Arrays}

The analysis context initializes array objects according to the specialization class declaration, making use of the fact that JSpec does not differentiate between array indices, so initializing a single index of the array creates the right context. The specialization context reads the contents of the array from the values file. The guards generated for an array object inspect the length and each index of the array, as required by the predicate. 


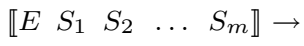

$\llbracket E \rrbracket \llbracket S_{-} 1 \rrbracket \ldots \llbracket S_{-} m \rrbracket$

$\llbracket$ specclass $E$ specializes $J\left\{\begin{array}{lllll}p_{1} & p_{2} & \ldots & p_{k} & e\end{array}\right\} \rrbracket \rightarrow$

public aspect $E\{$

public boolean $J$.guard_E $(\mathcal{P} \llbracket e \rrbracket)\{$

$\mathcal{G} \llbracket p_{1} \rrbracket$ (this.) $\mathcal{G} \llbracket p_{2} \rrbracket$ (this.) $\ldots \mathcal{G} \llbracket p_{k} \rrbracket$ (this.) $\quad \mathcal{G} \llbracket e \rrbracket(\epsilon)$

return true;

\}

pointcut entrypoint $\left(J \_j, \mathcal{P} \llbracket e \rrbracket\right)$ :

call (int $R . f\left(T_{1}, T_{2}, \ldots, T_{h}\right)$ )

$\& \& \operatorname{args}\left(r_{1}, r_{2}, \ldots, r_{h}\right)$

$\& \& \operatorname{target}(r)$;

int $\operatorname{around}\left(R \_r, T_{1} r_{1}, T_{2} r_{2}, \ldots, T_{h} r_{h}\right)$ :

entrypoint $\left(\_r, r_{1}, r_{2}, \ldots, r_{h}\right)\{$

if ( $r$.guard_L $L\left(r_{1}, r_{2}, \ldots, r_{h}\right)$

return $\bar{j} . f \operatorname{spec}\left(r_{1}, r_{2}, \ldots, r_{h}\right)$;

\} else \{

\}

return proceed $\left(\_R, r_{1}, r_{2}, \ldots, r_{h}\right)$;

\}

\}

where $e=$ public int $f\left(T_{1} r_{1}, T_{2} r_{2}, \ldots, T_{h} r_{h}\right)$ \{where $\left.\ldots\right\}$

$r=\operatorname{lowercase}(R)$ and $j=$ lowercase $(J)$

$\llbracket$ specclass $S_{i}$ specializes $J_{i}\left\{p_{1} p_{2} \ldots p_{k} \quad e\right\} \rrbracket \rightarrow$

public boolean $J_{i} i$._guard_ $S_{i}()\{$

$\mathcal{G} \llbracket p_{1} \rrbracket($ this. $) \quad \mathcal{G} \llbracket p_{2} \rrbracket($ this. $) \ldots \mathcal{G} \llbracket p_{k} \rrbracket$ (this.)

return true;

\}

$\mathcal{P} \llbracket$ public int $f\left(T_{1} r_{1}, T_{2} r_{2}, \ldots, T_{h} r_{h}\right)\left\{\right.$ where $p_{1} \ldots$ where $\left.p_{k}\right\} \rrbracket \rightarrow$ $T_{1} r_{1}, T_{2} r_{2}, \ldots, T_{h} r_{h}$

Figure 14: Top-level declarations for guards

$\mathcal{G} \llbracket v==$ number $\rrbracket(p) \rightarrow$ if $(!(p v==$ number $))$ return false;

$\mathcal{G} \llbracket v: J \rrbracket(p) \rightarrow$ if $(!(p v$.getClass ()$==J$.class $))$ return false;

$\mathcal{G} \llbracket v: S \rrbracket(p) \rightarrow$ if $\left(!\left(p v \cdot \operatorname{get} C\right.\right.$ lass ()$==R_{S}$.class $\left.)\right)$ return false; if $\left(!\left(\left(\left(R_{S}\right) p v\right)\right.\right.$._guard $\left.\left.S()\right)\right)$ return false;

where $R_{S}=$ rootclass $(S)$

$\mathcal{G} \llbracket$ public int $f\left(T_{1} r_{1}, T_{2} r_{2}, \ldots, T_{h} r_{h}\right)\left\{\right.$ where $p_{1} \ldots$ where $\left.p_{l}\right\} \rrbracket(p) \rightarrow$ $\mathcal{G} \llbracket p_{1} \rrbracket(p) \quad \mathcal{G} \llbracket p_{2} \rrbracket(p) \quad \ldots \mathcal{G} \llbracket p_{k} \rrbracket(p)$

Figure 15: Translation of predicates for guards 


\begin{tabular}{|c|c|c|c|c|c|c|c|c|c|c|}
\hline \multirow[b]{2}{*}{ Experiment } & \multicolumn{5}{|c|}{ IBM JIT 1.3 .1} & \multicolumn{5}{|c|}{ Sun Hotspot 1.4 -server } \\
\hline & $T_{\mathrm{G}}$ & $T_{\mathrm{SI}}$ & speedup & $T_{\mathrm{SO}}$ & speedup & $T_{\mathrm{G}}$ & $T_{\mathrm{SI}}$ & speedup & $T_{\mathrm{SO}}$ & speedup \\
\hline Power & 747 & 653 & $14 \%$ & 168 & $345 \%$ & 1343 & 319 & $321 \%$ & 168 & $700 \%$ \\
\hline Controller & 1498 & 1012 & $48 \%$ & $\dagger$ & $\dagger$ & 1528 & 1036 & $47 \%$ & $\dagger$ & $\dagger$ \\
\hline Arith-Int & 1036 & 653 & $-3 \%$ & 62 & $1571 \%$ & 1259 & 1077 & $17 \%$ & 506 & $149 \%$ \\
\hline OoLaLa & 1538 & 712 & $116 \%$ & 648 & $137 \%$ & 1314 & 863 & $52 \%$ & 894 & $47 \%$ \\
\hline
\end{tabular}

( $\dagger$ : The context varies with each iteration, so only $T_{\mathrm{SI}}$ can be measured.)

Table 1: Experimental results, times are real-time measured in milliseconds

\section{Related Work}

The specialization classes language and compiler presented by Volanschi et al. are the basis of our work [24]. The main limitation of specialization classes is the lack of support for specifying precise invariants over reference types, which is a core feature of Pesto. Other limitations include the lack of predicates on method parameters and the lack of proper support for off-line specialization. Conversely, the specialization classes compiler supports modification-time guards, which in many cases are more efficient than the call-time guards offered by Pesto (but which suffer from other limitations, as discussed in Section 4.4). Moreover, specialization classes support a notion of inheritance which can be used to define a precedence between entry points; we expect that a similar feature can be used in a future version of Pesto. Last, we note that Pesto is the only system of the two to have been completely integrated with a partial evaluator, and that the use of aspect-oriented programming in Pesto is an improvement over the parser/prettyprinter-based approach of specialization classes.

The specialization modules language of Le Meur et al. can be seen as a version of specialization classes for a modular version of $\mathrm{C}[13,17]$. Compared to Pesto, the main advantage of specialization modules is that binding-time declarations are checked during binding-time analysis, so that an analysis error is generated if the declared binding times are inconsistent with the derived binding times. Moreover, the tool support is much more mature, simplifying the use of partial evaluation as a configuration tool. On the other hand, specialization modules do not offer automatic generation of guards, and it is not possible to specify complex specialization contexts with aliasing such as the one required for the arithmetic interpreter. Nonetheless, specialization modules are integrated with Tempo, so an interesting improvement of Pesto would be to generate specialization modules that could be used in JSpec, thus unifying the two approaches.

\section{Conclusion and Future Work}

The Pesto language allows precise declaration of specialization scenarios for programs written in an object-oriented style of programming. The implementation is integrated with the JSpec partial evaluator, and automatically generates 
both the context information needed for the specialization of Java programs and guards that select the specialized code in the appropriate context. In our experience, these features dramatically improve the ease with which a partial evaluator such as JSpec can be used. We believe that declarative front-ends should be considered an essential part of any partial evaluator.

In terms of future work, we are interested in generalizing Pesto by factorizing the JSpec-specific features of the compiler into a pluggable back-end. The current approach of relying on a default constructor and public fields would then simply be one available back-end. Alternate back-ends could use Java reflection, AspectJ, or an interface specific to the partial evaluator which e.g. allows access to private fields (such an interface is under development for JSpec).

\section{References}

[1] H. Markmann Andersen. Deklarativ specialisering af objektorienterede sprog. Master's thesis, DAIMI, University of Aarhus, May 2003.

[2] L.O. Andersen. Program Analysis and Specialization for the C Programming Language. PhD thesis, Computer Science Department, University of Copenhagen, May 1994. DIKU Technical Report 94/19.

[3] R. Baier, R. Glück, and R. Zöchling. Partial evaluation of numerical programs in Fortran. In ACM SIGPLAN Workshop on Partial Evaluation and Semantics-Based Program Manipulation (PEPM'94), pages 119-132, Orlando, FL, USA, June 1994. Technical Report 94/9, University of Melbourne, Australia.

[4] A. Bondorf. Self-Applicable Partial Evaluation. PhD thesis, DIKU, University of Copenhagen, Denmark, 1990. Revised version: DIKU Report $90 / 17$.

[5] C. Consel. A tour of Schism: a partial evaluation system for higher-order applicative languages. In Partial Evaluation and Semantics-Based Program Manipulation (PEPM'93), pages 66-77, Copenhagen, Denmark, June 1993. ACM Press.

[6] C. Consel, L. Hornof, F. Noël, J. Noyé, and E.N. Volanschi. A uniform approach for compile-time and run-time specialization. In O. Danvy, R. Glück, and P. Thiemann, editors, Partial Evaluation, International Seminar, Dagstuhl Castle, number 1110 in Lecture Notes in Computer Science, pages 54-72, Dagstuhl Castle, Germany, February 1996. Springer-Verlag.

[7] E. Gamma, R. Helm, R. Johnson, and J. Vlissides. Design Patterns: Elements of Reusable Object-Oriented Software. Addison-Wesley, 1994.

[8] L. Hornof, J. Noyé, and C. Consel. Effective specialization of realistic programs via use sensitivity. In P. Van Hentenryck, editor, Proceedings of the Fourth International Symposium on Static Analysis (SAS' $\left.{ }^{\prime} 7\right)$, volume 
1302 of Lecture Notes in Computer Science, pages 293-314, Paris, France, September 1997. Springer-Verlag.

[9] IBM. IBM JDK 1.3.1, 2001. Accessible from http://www.ibm.com/java/jdk.

[10] N.D. Jones, C. Gomard, and P. Sestoft. Partial Evaluation and Automatic Program Generation. International Series in Computer Science. PrenticeHall, June 1993.

[11] G. Kiczales, E. Hilsdale, J. Hugunin, M. Kersten, J. Palm, and W. Griswold. An overview of AspectJ. In J.L. Knudsen, editor, Proceedings of the European Conference on Object-Oriented Programming (ECOOP'01), volume 2072 of Lecture Notes in Computer Science, pages 327-353, Budapest, Hungary, 2001.

[12] G. Kiczales, J. Lamping, A. Mendhekar, C. Maeda, C. Lopes, J. Loingtier, and J. Irwin. Aspect-oriented programming. In M. Aksit and S. Matsuoka, editors, Proceedings of the European Conference on Object-oriented Programming (ECOOP'97), volume 1241 of Lecture Notes in Computer Science, pages 220-242, Jyväskylä, Finland, June 1997. Springer.

[13] A.F. Le Meur, J.L. Lawall, and C. Consel. Towards bridging the gap between programming languages and partial evaluation. In Proceedings of the 2002 ACM SIGPLAN workshop on Partial evaluation and semanticsbased program manipulation, pages 9-18. ACM Press, 2002.

[14] J.W. Lloyd and J.C. Shepherdson. Partial evaluation in logic programming. Journal of Logic Programming, 11:217-242, 1991.

[15] M. Luján. Object oriented linear algebra. Master's thesis, University of Manchester, December 1999.

[16] M. Luján, T.L. Freeman, and J.R. Gurd. OoLALA: an object oriented analysis and design of numerical linear algebra. In M.B. Rosson and D. Lea, editors, OOPSLA'00 Conference Proceedings, ACM SIGPLAN Notices, pages 229-252, Minneapolis, MN USA, October 2000. ACM Press, ACM Press.

[17] A.F Le Meur, J.L. Lawall, and C. Consel. Specialization scenarios: A pragmatic approach to declaring program specialization. Higher-Order and Symbolic Computation. To appear.

[18] U.P. Schultz. Object-Oriented Software Engineering Using Partial Evaluation. PhD thesis, University of Rennes I, Rennes, France, December 2000.

[19] U.P. Schultz. Partial evaluation for class-based object-oriented languages. In O. Danvy and A. Filinski, editors, Symposium on Programs as Data Objects II, volume 2053 of Lecture Notes in Computer Science, pages 173197, Aarhus, Denmark, May 2001. 
[20] U.P. Schultz, J. Lawall, C. Consel, and G. Muller. Towards automatic specialization of Java programs. In R. Guerraoui, editor, Proceedings of the European Conference on Object-oriented Programming (ECOOP'99), volume 1628 of Lecture Notes in Computer Science, pages 367-390, Lisbon, Portugal, June 1999. Springer-Verlag.

[21] U.P. Schultz, J.L. Lawall, and C. Consel. Automatic program specialization for Java. TOPLAS, 25:452-499, July 2003.

[22] U.P. Schultz, J.L. Lawall, C. Consel, and G. Muller. Specialization patterns. In Proceedings of the $15^{\text {th }}$ IEEE International Conference on Automated Software Engineering (ASE 2000), pages 197-206, Grenoble, France, September 2000. IEEE Computer Society Press.

[23] E.N. Volanschi. Une approche automatique à la spécialisation de composants système. Thèse de doctorat, University of Rennes I, February 1998.

[24] E.N. Volanschi, C. Consel, G. Muller, and C. Cowan. Declarative specialization of object-oriented programs. In OOPSLA'97 Conference Proceedings, pages 286-300, Atlanta, GA, USA, October 1997. ACM Press.

[25] AspectJ home page, 2000. Accessible from http://aspectj.org. Xerox Corp. 


\section{A Pesto grammar}

pesto_file
specialization_class
predicate_declaration
method_declaration
variable_declaration
parameter_declaration
value_declaration
type_declaration
array_type
array_index
types
type

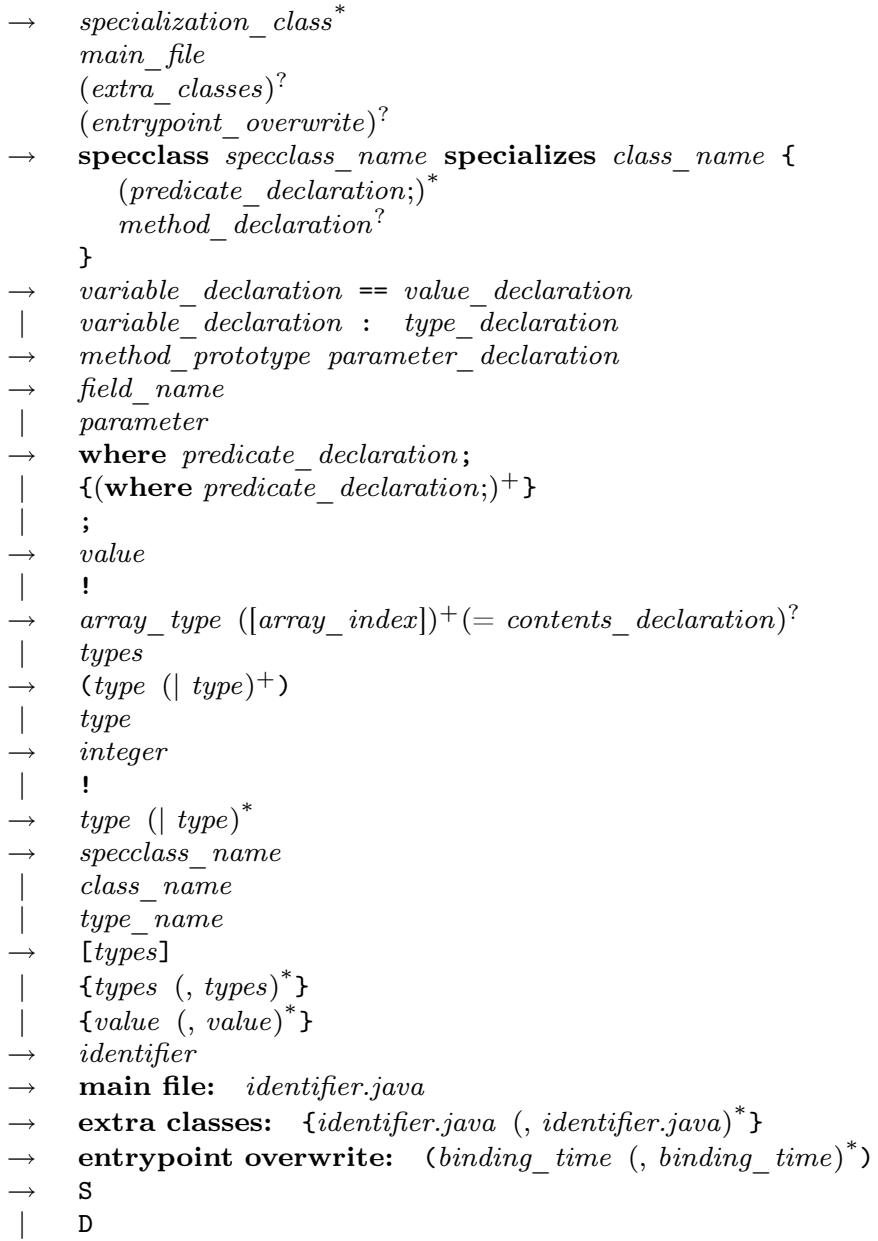

